\title{
Single Cell Multiplex Protein Measurements through Rare Earth Element Immunolabeling, Laser Capture Microdissection and Inductively Coupled Mass Spectrometry
}

\author{
Amir Liba ${ }^{1}$ and Jonathan Wanagat ${ }^{2 *}$ \\ ${ }^{1}$ Agilent Technologies, USA \\ ${ }^{2}$ Department of Medicine, Division of Geriatrics, UCLA, Los Angeles, CA 90095, USA
}

"Corresponding author: Jonathan Wanagat, Department of Medicine, Division of Geriatrics, University of California, Los Angeles, 900 Veteran Ave, Suite 24-188, Los Angeles, CA 90095, USA, Tel: 310-825-8253, E-mail: jwanagat@mednet.ucla.edu

Rec date: Oct 02, 2014, Acc date: Oct 28, 2014, Pub date: Oct 30, 2014

Copyright: @ 2014 Liba A, et al. This is an open-access article distributed under the terms of the Creative Commons Attribution License, which permits unrestricted use, distribution, and reproduction in any medium, provided the original author and source are credited.

\begin{abstract}
Complex diseases such as heart disease, stroke, cancer, and aging are the primary causes of death in the US. These diseases cause heterogeneous conditions among cells, conditions that cannot be measured in tissue homogenates and require single cell approaches. Understanding protein levels within tissues is currently assayed using various molecular biology techniques (e.g., Western blots) that rely on milligram to gram quantities of tissue homogenates or immunofluorescent (IF) techniques that are limited by spectral overlap. Tissue homogenate studies lack references to tissue structure and mask signals from individual or rare cellular events. Novel techniques are required to bring protein measurement sensitivity to the single cell level and offer spatiotemporal resolution and scalability. We are developing a novel approach to protein quantification by exploiting the inherently low concentration of rare earth elements (REE) in biological systems. By coupling REE-antibody immunolabeling of cells with laser capture microdissection (LCM) and ICP-QQQ, we are achieving multiplexed protein measurement in histological sections of single cells. This approach will add to evolving single cell techniques and our ability to understand cellular heterogeneity in complex biological systems and diseases.
\end{abstract}

Keywords: Laser capture microdissection; Inductively coupled mass spectrometry; Rare earth elements

\section{Introduction}

Our interest in developing an approach for single cell protein measurement grew out of our ongoing studies of focal mitochondrial defects in aging skeletal muscle [1]. Aging is the primary risk factor for the primary causes of death in the United States [2] and like many complex biological processes and diseases such as cancer, heart disease, and neurodegeneration, aging occurs heterogeneously at the cellular and tissue level [3-6]. The focal nature of these processes is reflected in a variety of histopathological changes such as fatty infiltration, fiber atrophy, inflammation and fibrosis. Tissue homogenate studies lack a reference to structural changes and suppress signals from unique, rare events. The heterogeneous distribution of damage necessitates the use of single cell approaches that provide increased sensitivity, spatial resolution, multiplexing and scalability.

Traditional approaches to protein measurement include Western blots, enzyme-linked immunosorbent assay (ELISA), mass spectrometry and immunofluorescence [7]. Approaches such as Western blots, mass spectrometry and ELISA require protein fractions from tissue homogenates that typically require gram to milligram tissue quantities. The homogenization procedures destroy any spatial resolution inherent to the original tissue samples and the tissue amounts required result in the sampling of millions of individual cells. Thus, protein signals from cells in a unique local environment or cells undergoing a rare phenomenon (e.g., malignant transformation, apoptosis, senescence) are lost in the background. Immunofluorescent approaches, such as confocal microscopy provide spatial resolution, but the measurement of multiple proteins within a pathway is limited by the spectral overlap of the fluorophores [8].

A recent approach used REE not normally found in biological systems as antibody tags in atomic mass spectrometric analysis of single hematopoetic cells - termed single cell "mass cytometry" [9]. Using this approach, 34 parameters were analyzed simultaneously in single cells. The use of heavy-metal immunolabeling has a number of distinct advantages to immunohistochemistry (IHC) and IF approaches including opportunities for absolute quantitation, lack of signal overlap, complete lack of "auto-fluorescence" or background and adaptability to high-throughput analyses.

We have adapted the mass cytometry approach for use in histological tissue sections by using laser capture microdissection (LCM) to isolate defined numbers of REE-antibody-labeled cells. This approach allows analysis of single or multiple targeted cells from tissue samples of solid organs and archived pathological specimens. Coupled with single cell DNA and RNA methods, this approach will add to elucidating cell-to-cell variation and rare biochemical conditions, which may have important consequences in complex biological pathways and diseases.

\section{Materials and Methods}

\section{Animals and tissue collection}

Male C57BL/6 mice were purchased from the National Institute on Aging colony at six months of age. We chose male mice to allow us to compare our LCM-ICP-QQQ measurements to our previous single 
cell studies in male mouse tissues. Mice were given free access to water and food and euthanized by carbon dioxide inhalation with death confirmed by usual methods. The quadriceps femoris muscles were dissected from each animal, embedded in OCT mounting media (Miles Inc.) and frozen in liquid nitrogen. Samples were stored at $-80^{\circ} \mathrm{C}$ until sectioned. All animal experiments were approved by the UCLA Institutional Animal Care and Use Committee.

\section{Tissue staining with REE}

Mouse quadriceps femoris muscle samples were raised to sectioning temperature of $-16^{\circ} \mathrm{C}$. Ten micron thick sections were obtained and placed on Arcturus PEN membrane glass microscope slides (Life Technologies). Sections were stored at $-80^{\circ} \mathrm{C}$ until immunolabeling was performed. REE labeled antibodies were prepared and purified according to the manufacturer's instructions (DVS Sciences). Antimouse fast myosin antibody (MY-32, Sigma) was tagged with 141Pr. Anti-mouse GAPDH antibody (G9545, Sigma) was tagged with 171Yb.
Anti-mouse interleukin-6 (IL-6) antibody was purchased as already tagged with 167Er from the manufacturer (DVS Sciences). MAXPAR Nucleic Acid Intercalator (DVS Sciences) labeled with 103Rh was used to stain cell nuclei. Immunostaining was performed as described previously [1]. Stained slides were dehydrated quickly through an ethanol and xylene series and dried completely before LCM.

\section{Laser capture microdissection of individual muscle fibers}

Skeletal muscle fibers were captured using a Leica LMD7000 laser dissecting microscope. Dissections were done using the 20x objective with laser settings of power $=25$, aperture $=17$, speed $=10$, balance $=15$, head current $=100$ and pulse frequency $=800$. Single fibers were collected (Figure 1). Fibers were captured into individual wells of a 48well microslide. Each captured sample was visually inspected to confirm capture. Captured cells were digested with one microliter of $70 \%$ nitric acid and digested samples taken up in nine microliters of water. Samples were stored at $4^{\circ} \mathrm{C}$ until analysis by ICP-QQQ.
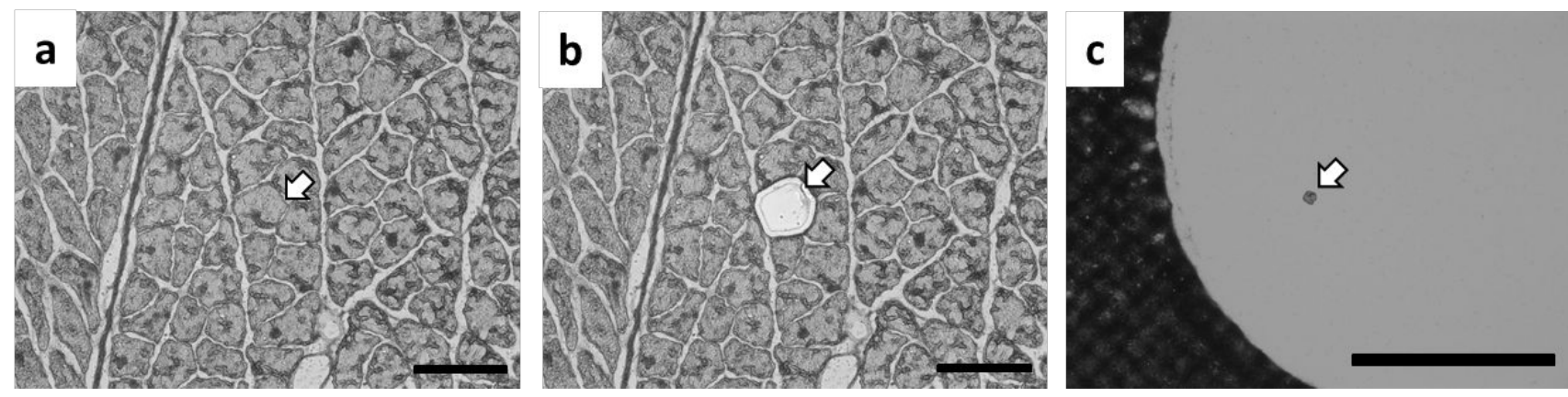

Figure 1: Laser capture microdissection of an individual skeletal muscle fiber before capture (a), the tissue section after capture (b) and the captured fiber (c) Arrow denotes the targeted fiber (a), the selective laser cutting of only the targeted fiber (b) and the captured fiber (c). Black bar is 50 microns ( $a$ and $b$ ) and 500 microns (c).

\section{ICP-QQQ of REE-immunolabeled muscle fibers}

To the Agilent 8800 ICP-QQQ, ten microliter samples were either injected via syringe pump or by an Agilent 1260 Cap-LC at a flow rate of $10 \mathrm{microliter/min}$. The ICP-QQQ was equipped with a low-flow nebulizer and a total consumption spray-chamber. Sensitivity and ion lens voltages were auto-tuned via MassHunter 4.1 software using 1 ppb tune solution (Agilent, DE). Typical sample introduction parameters were used; $1550 \mathrm{~W}$ RF power, $1 \mathrm{~L} / \mathrm{min}$ carrier gas, 0.15 $\mathrm{L} / \mathrm{min}$ dilution gas, $8 \mathrm{~mm}$ sampling depth, and analysis was performed in TRA mode with $0.2 \mathrm{sec}$ integration time. To ensure the entire sample was delivered to the ICP-QQQ, the injected samples were bracketed by air injection.

\section{Statistical analysis}

All data with normal distribution were presented as means \pm SEM calculated with GraphPad Prism software.

\section{Results}

\section{ICP-QQQ of REE and protein measurement}

Because of our interest in age-related changes to single muscle fibers, we applied the LCM-ICP-QQQ technique to histological sections of mouse skeletal muscle. We immunolabeled muscle sections with the three REE-labeled antibodies (i.e., antibodies against mouse myosin, IL-6 and GAPDH) and an REE-labeled DNA intercalator. We used laser microdissection to capture five randomly selected muscle fibers from the immunolabeled section and a representative fiber capture is shown in Figure 1. Each captured fiber was digested and analyzed as described in Materials and Methods. As demonstrated in Figure 1, the LCM approach facilitates the capture of a single cell without contamination by surrounding cells and the ability to confirm cell capture before further analysis on the ICP-QQQ.

The Agilent 8800 ICP-QQQ measured the REE signal from each labeled antibody and the DNA intercalator in individual muscle fibers captured by LCM. Table 1 shows the ICP-QQQ data from five representative single muscle fibers. Highest counts were detected for $141 \mathrm{Pr}$ and 103Rh that correspond to single cell myosin and DNA content, respectively, while lower counts were detected for 167Er and $171 \mathrm{Yb}$ that correspond to single cell IL- 6 and GAPDH content. The presence of REE in biological samples is extremely low, resulting in negligible ICP-QQQ background from REE. The average signal to noise ratio across the five samples and the four REE tags in Table 1 was 85. Negative controls included muscle fibers laser captured from mouse muscle sections that were not immunolabeled with REEs and these showed no detectable signals (data not shown). 


\section{Discussion}

We demonstrate a novel approach for multiplex protein measurements in single cells isolated from histological sections. Our initial data, shown in Table 1, demonstrate the ability to detect REE tagged antibodies for three different proteins and an REE tagged DNA intercalator in single mouse muscle cells. The higher counts for the $141 \mathrm{Pr}$ and 103Rh correspond to the expected higher content of myosin, a primary component of the sarcomere, and DNA in the nuclei of these multinucleate cells as compared to the lower counts for $167 \mathrm{Er}$ and $171 \mathrm{Yb}$ representing IL-6 and GAPDH, respectively, which are known to be present at lower levels in these cells. The variability in $141 \operatorname{Pr}$ (myosin) signal is likely due to fiber size differences as is evident in Figure 1, while the variability in the $103 \mathrm{Rh}$ (DNA) signal is likely due to differences in nuclei number between different cells. We are developing protocols to normalize the REE counts to cell size or other housekeeping proteins as is routinely done with protein detection in Western blots.

\begin{tabular}{|l|l|l|l|l|l|l|}
\hline $\begin{array}{l}\text { REE } \\
\text { label }\end{array}$ & Fiber 1 & Fiber 2 & Fiber 3 & Fiber 4 & Fiber 5 & Average counts $^{\mathbf{b}}$ \\
\hline $\begin{array}{l}{ }^{141} \mathrm{Pr}-\mathrm{Ab}- \\
\text { Myosin }\end{array}$ & $2562^{\mathrm{a}}$ & 2726 & 2835 & 3198 & 1976 & $2659 \pm 200$ \\
\hline $\begin{array}{l}167 \mathrm{Er}-\mathrm{Ab}- \\
\text { IL6 }\end{array}$ & 178 & 273 & 345 & 299 & 233 & $266 \pm 28$ \\
\hline $\begin{array}{l}171 \mathrm{Yb}-\mathrm{Ab}- \\
\text { GAPDH }\end{array}$ & 105 & 126 & 160 & 138 & 110 & $127 \pm 10$ \\
\hline $\begin{array}{l}\text { 103Rh- } \\
\text { DNA }\end{array}$ & 877 & 712 & 1482 & 776 & 1132 & $996 \pm 141$ \\
\hline
\end{tabular}

aData are represented as counts for each respective mass analyzed $(103,141$, 167, and $171 \mathrm{AMU}$ ).

${ }^{b}$ Average counts for each REE label \pm SEM.

Table 1: ICP-QQQ data from single, REE-labeled muscle fibers.

The recently published approach of single cell "mass cytometry" is currently limited to the study of cells in suspension [9]. LCM allows application of this approach to a wider range of tissue samples and standard tissue sections. These sections can be from any type of pathological samples including biopsies and fine-needle aspirations. The use of LCM also provides access to archived formalin-fixed, paraffin-embedded tissues. We used a standard tissue section immunolabeling protocol for the antibodies and DNA intercalator in this study and this protocol may need to be optimized for each antibody as would be done for standard immunolabeling. Because the REE tagging does not interfere with standard immunofluorescent or immonhistochemical secondary labeling or detection, antibody validation protocols do not need to be altered. With the availability of 32 unique REE labeling kits and the ability of generating different panels of antibodies, there is extensive capacity for multiplex protein measurement. We demonstrated only three antibody labels and one DNA intercalator, but are expanding this to higher levels of multiplexing.

Challenges with this approach include sample delivery, validation and scalability. We used microliter volumes of nitric acid or other buffers for cell lysis, which are loaded into the ICP-QQQ. Consideration must be given to non-specific binding of digested proteins to the sample introduction system, contamination between samples when loading sequential samples, and introduction of control or validation samples. The addition of blocking solutions and different mobile phases may prevent non-specific binding, which will likely be tissue specific. Similar steps must be taken to avoid cross-sample contamination when loading multiple samples through the same sample delivery system. Validation of the single cell ICP-QQQ results is challenging because most other single cell protein measurement approaches are semi-quantitative or qualitative. Specificity could be tested in cells types with known differential protein expression such as slow and fast twitch muscle fibers and antibodies for different myosin types in these fibers. Transgenic animals with known levels of target protein expression could be used to test sensitivity differences between biological samples [10]. With the study of single cells, scalability is important to facilitate building cell profiles from large numbers of single cells. We increased the LCM throughput with the use of a multiwell slide for capturing 48 individual samples for subsequent digestion and are assessing different methods for high-throughput ICP-QQQ sample delivery.

We are making a number of improvements to the LCM-ICP-QQQ approach. We are expanding our panel of labeled antibodies to allow us to examine more proteins in single cells. Panels are in development to examine entire cellular pathways (e.g., insulin signaling) or multicomponent protein complexes (i.e., the dystroglycan complex affected in muscular dystrophies). The laser capture approach facilitates the study of other tissue components in histological sections. In addition to single cells including focal areas of fibrosis, inflammation and fatty infiltration, niches not easily studied by homogenate approaches. LCM of organelles such as mitochondria [11] open the possibilities of subcellular protein measurement by the LCM-ICP-QQQ method.

Our approach to measure single cell protein content using REE immunolabeling, LCM and ICP-QQQ is combining standard and emerging techniques to facilitate studies in a wide variety of biological samples at the single cell level. With other single cell analyses, this approach will help uncover heterogeneous cellular events in complex diseases and add to an integrated view of the underlying pathology.

\section{Acknowledgements}

This work was supported by the American Federation for Aging Research, the UCLA Hartford Center of Excellence; NIH awards K08 AG032873, P30 AG028748, P30 AR057230 and an Ellison Medical Foundation New Scholar Award to Dr. Wanagat. Laser capture microdissection was performed at the CNSI Advanced Light Microscopy/Spectroscopy Shared Resource Facility at UCLA, supported with funding from NIH-NCRR shared resources grant (CJX1-443835-WS-29646) and NSF Major Research Instrumentation grant (CHE-0722519).

\section{References}

1. Wanagat J, Cao Z, Pathare P, Aiken JM (2001) Mitochondrial DNA deletion mutations colocalize with segmental electron transport system abnormalities, muscle fiber atrophy, fiber splitting, and oxidative damage in sarcopenia. Faseb J 15: 322-332.

2. Butler RN, Miller RA, Perry D, Carnes BA, Williams TF, et al. (2008) New model of health promotion and disease prevention for the 21st century. BMJ (Clinical research ed 337: a399.

3. Jaitin DA, Kenigsberg E, Keren-Shaul H, Elefant N, Paul F, et al. (2014) Massively parallel single-cell RNA-seq for marker-free decomposition of tissues into cell types. Science 343: 776-779.

4. Morrison CD, Liu P, Woloszynska-Read A, Zhang J, Luo W, et al. (2014) Whole-genome sequencing identifies genomic heterogeneity at a 
Citation: Liba A, Wanagat J (2014) Single Cell Multiplex Protein Measurements through Rare Earth Element Immunolabeling, Laser Capture Microdissection and Inductively Coupled Mass Spectrometry. J Cytol Histol 5: 290. doi:10.4172/2157-7099.1000290

Page 4 of 4

nucleotide and chromosomal level in bladder cancer. Proceedings of the National Academy of Sciences of the United States of America 111: E672-681.

5. Li Y, Guo X, Xue Q, Zhu M, Gao L, et al. (2013) Single cell gene profiling revealed heterogeneity of paracrine effects of bone marrow cells in mouse infarcted hearts. PLoS One 8: e68270.

6. Gerschutz A, Heinsen H, Grunblatt E, Wagner AK, Bartl J, et al. (2014) Neuron-Specific Alterations in Signal Transduction Pathways associated with Alzheimer's Disease. J Alzheimers Dis 40: 135-142.

7. Kingsmore SF (2006) Multiplexed protein measurement: technologies and applications of protein and antibody arrays. Nat Rev Drug Discov 5 310-320.
8. Perfetto SP, Chattopadhyay PK, Roederer M (2004) Seventeen-colour flow cytometry: unravelling the immune system. Nat Rev Immunol 4: 648-655.

9. Bendall SC, Simonds EF, Qiu P, Amir el-AD, Krutzik PO, et al. (2011) Single-cell mass cytometry of differential immune and drug responses across a human hematopoietic continuum. Science 332: 687-696.

10. Marshall JL, Holmberg J, Chou E, Ocampo AC, Oh J, et al. (2012) Sarcospan-dependent Akt activation is required for utrophin expression and muscle regeneration. J Cell Biol 197: 1009-1027.

11. Pflugradt R, Schmidt U, Landenberger B, Sänger T, Lutz-Bonengel S (2011) A novel and effective separation method for single mitochondria analysis. Mitochondrion 11: 308-314. 